\title{
Dimensiones Clave para el Desarrollo Escolar Inclusivo: Conocimiento Emancipador en una Experiencia de Asesoramiento Colaborativo
}

\author{
Key Dimensions for the Inclusive Educational Development: \\ Emancipatory Knowledge in a Collaborative Counselling \\ Experience
}

\author{
Ignacio Figueroa-Céspedes * \\ Jorge Soto Cárcamo \\ Cristopher Yáñez-Urbina \\ Universidad Diego Portales, Chile
}

\begin{abstract}
El presente artículo analiza las claves para la puesta en marcha de un proceso de desarrollo escolar inclusivo a partir de un conjunto de experiencias de asesoramiento colaborativo, desarrolladas entre los años 2014 y 2017 en la ciudad de Santiago de Chile, utilizando los materiales de la Guía para la Inclusión Educativa. Se procedió por medio de un estudio cualitativo que sistematiza una serie de indagaciones realizadas desde un diseño de investigación-acción participativa en conjunto con comunidades educativas, las técnicas de producción de información fueron notas de campo, materiales de trabajo producidos por los participantes, entrevistas y registros de reuniones que fueron sometidos a un análisis de contenido y a una triangulación de datos. Los resultados dan cuenta de cinco dimensiones claves, a saber: culturas colaborativas, investigación-acción participativa, liderazgo inclusivo, visión de cambio integral y asesoramiento colaborativo. Se concluye que las dimensiones son co-dependientes y articuladas para orientar el afrontamiento de los procesos de cambio inclusivo. Finalmente, existe un conjunto de situaciones en las cuales el asesoramiento colaborativo puede sortear un complejo escenario marcado por las exigencias de estándares de calidad y la valoración a diversidad, por medio de un abordaje centrado en el proceso.
\end{abstract}

Descriptores: Justicia social; Cooperación educacional; Democratización de la educación; Investigación aplicada.

This article analyzes the keys for the implementation of an inclusive school development process from a set of collaborative counseling experiences, developed between 2014 and 2017 in the city of Santiago de Chile, using the materials of the Guide for Educational Inclusion. We proceeded by means of a qualitative study that systematizes a series of inquiries carried out from a participatory action research design in conjunction with educational communities, the information production techniques were field notes, work materials produced by the participants, interviews and records of meetings that were subjected to a content analysis and data triangulation. The results reveal five key dimensions, namely: collaborative cultures, participatory action research, inclusive leadership, comprehensive change vision, and collaborative mentoring. It is concluded that the dimensions are co-dependent and articulated to guide the coping of inclusive change processes. Finally, there is a set of situations in which collaborative counseling can overcome a complex scenario marked by the demands of quality standards and the valuation of diversity, through a process-centered approach.

Keywords: Social justice; Educational cooperation; Democratization of education; Applied research.

*Contacto: ignacio.figueroa@mail.udp.cl

ISSN: 0718-7378

www.rinace.net/rlei/
Recibido: 29/04/2021

$1^{a}$ Evaluación: 20/08/2021

Aceptado: 08/10/2021 


\section{Revisión de la literatura}

Actualmente, el desarrollo de políticas educativas se encuentra marcado por la yuxtaposición de dos visiones antagónicas. De un lado, la preocupación por la eficacia escolar, encarnada principalmente en el desarrollo de indicadores estandarizados (Galaz et al., 2019; Herrera et al., 2018). Mientras que, por el otro, un énfasis por el reconocimiento de la diversidad educativa desde el marco de la inclusión educativa y la educación intercultural (Tomé y Manzano, 2016).

En Chile estas cuestiones se han plasmado en múltiples dificultades en el quehacer educativo de las escuelas. Una de ellas es la constante presión que experimentan los equipos por responder a indicadores estandarizados de calidad que fomentan la competitividad entre establecimientos y, al interior de ellos, entre estudiantes derivando en la exclusión de aquellos que no logran cumplir con los estándares (Sisto, 2020). Lo anterior redunda en la adscripción a una gestión de la diversidad desde el modelo del déficit, priorizando el etiquetamiento individual (Apablaza, 2018).

Por otro lado, se suma la sintomática falta de tiempo que tienen los equipos pedagógicos y de apoyo para planificar, colaborar y repensar sus prácticas de enseñanza (Urbina, et al. 2017), hecho que fomenta el trabajo desde la intuición del profesor más que desde su profesionalidad lo que afecta la revisión crítica de sus prácticas en la escuela.

Frente a este escenario, y siguiendo a Ainscow (2005) se propone la herramienta de la indagación colaborativa como una "palanca de cambio" que permite re-pensar y transformar las instituciones educativas desde un marco de investigación-acción participativa, conectando con un foco en la construcción de propuestas de desarrollo inspiradas en valores y principios, que amplíen el bagaje de experiencias de cada establecimiento educativo (Booth y Ainscow, 2012; Figueroa-Céspedes et al., 2019) siendo una función política de la escuela para la sociedad (Dussel, 2004).

No obstante, la tensión entre los resultados estandarizados y la valoración de la diversidad produce una serie de dinámicas que pueden tanto entorpecer como facilitar los proyectos para el desarrollo escolar inclusivo. Siendo así, pueden variar dinámicamente entre mostrarse como plenamente eficientes u operar desde un plano meramente burocrático, así como también concebir las problemáticas de manera individualizada con un modelo deficitario o por medio de un enfoque de tipo comunitario (Figueroa-Céspedes et al., 2019).

De tal manera, en el presente artículo analizamos las claves para la puesta en marcha de un proceso de desarrollo escolar inclusivo a partir de la sistematización y triangulación de un conjunto de experiencias de asesoramiento colaborativo, desarrolladas entre los años 2014 y 2017, utilizando los materiales de la Guía para la Inclusión Educativa (Booth y Ainscow, 2012). Lo anterior implica dar cuenta del desafío asociado a la construcción de escuelas abiertas a la diversidad, desde procesos de deliberación colaborativa que rescatan el conocimiento local sobre la propia escuela para transformarla en conocimientoemancipación (Chavarría y García, 2004) visibilizando las voces que se encuentran silenciadas en los espacios educativos (Figueroa-Céspedes y Yáñez-Urbina, 2020) a través de la construcción de diálogos con los diversos actores (Long, 2007).

\subsection{Conocimiento emancipador}

Siguiendo a Figueroa-Céspedes y Yáñez-Urbina (2020), la noción de conocimiento emancipador guarda relación con una pregunta de orden ético sobre la educación: ¿Quién 
puede hablar sobre la escuela? o ¿Quién está autorizado a producir un conocimiento sobre la escuela, sus relaciones, dinámicas, problemas y sus posibilidades de cambio? Desde este enfoque cobra relevancia la cultura, entendida como un campo abierto y dinámico de pugnas, diálogos, luchas y alianzas existentes entre el amplio abanico de significados sobre el mundo (García Canclini, 2007) que conviven en el espacio educativo. Hablamos de una construcción colectiva de sentidos, saberes, creencias y valores que sostienen un sentimiento de inclusión social sobre aquello que llamamos realidad (Calderón, 2015) y que, en la actualidad, se encuentra cooptada en las instituciones escolares por formas de saber que apelan a la monoculturalidad (Booth y Ainscow, 2012; Dietz, 2012) invisibilizando la diversidad de visiones de mundo que la habitan (Ferrao, 2010).

Por lo tanto, al instalarse la cultura como un ámbito relevante en el campo de las prácticas cotidianas y su interrelación la vida de los sujetos (Hall, 2006) emerge el interés por la transformación de las relaciones que en ellas se ejercen (Rosas, 2012), encontrando su pleno sentido político por centrarse en una pugna de poder y en el diálogo entre actores (Long, 2007). En otras palabras, es una noción que pone en la palestra la necesidad de un proceso de re-culturación (Fullan, 2002) que cuestione y transforme la tradicional cultura escolar considerando la construcción y reflexión desde un marco ético-valórico inclusivo (Booth, 2005), superando problemáticas tales como la exclusión de la diferencia y las culturas de procedencia del estudiantado y el resto de la comunidad (Jorquera, 2015), un marcado adultocentrismo (Duarte, 2012), la proliferación de ambientes violentos (Abramovay, 2005), entre muchas otras.

En estas coordenadas, el desafío es tributario de las epistemologías feministas que se preguntan por las vidas que importan (Butler, 2006), su relación con el enfoque poscolonial (Spivak, 2011) y desde el plano de la justicia cognitiva (Santos, 2018). De tal manera, se instaura una noción de crítica en contra de un sujeto cognoscente universalista y que ha adoptado el rol de testigo modesto o legítimo interlocutor de la objetividad (Haraway, 2004) tanto para hablar como para representar a otros, mientras los mantiene silenciados como sujetos interdictos y sin una agencia política directa (Haraway, 1999).

En suma, resulta relevante proponer un abordaje centrado en las culturas en la escuela como una amplia diversidad cultural que no se limita al colectivo de estudiantes inmigrantes o pertenecientes a pueblos indígenas (Ferrao, 2010), sino que se extiende a todos los miembros de la comunidad educativa desde el estudiantado, el personal no docente, familias, hasta el equipo directivo y los profesores (Stoll y Fink, 1999). La escuela entendida como una multiplicidad cultural, tiene el desafío de abrir espacios para la escucha y, por sobre todo, de prepararse para dar cabida a aquellas voces silenciadas (Figueroa-Céspedes y Yáñez-Urbina, 2020).

Siendo así, el conocimiento emancipador constituye una propuesta ético-política donde priman los procesos de diálogo, la negociación de recursos y significados generados de un movimiento de co-construcción de saberes locales a partir del trabajo con los diversos actores de la comunidad educativa (Long, 2007). Asimismo, implica un rechazo a una acción política basada en la representación (Haraway, 1999), en donde se instala una forma de verdad acabada y basada en la objetividad de algunos sujetos privilegiados, pero cuyo rol evidencia una serie de desigualdades que han sido normalizadas y naturalizadas (Haraway, 2004) en las instituciones escolares.

En su lugar, el conocimiento emancipador apuesta por un trabajo desde el enfoque de los conocimientos situados en tanto construcción de verdades desde la interacción, 
intercambio, discusión y problematización que considera la multiplicidad de aristas existentes con miras a la transformación de los contextos (Haraway, 1995). Dicho de otra forma, se basa en un movimiento que busca escuchar la subalternidad (Spivak, 2011) como un ejercicio práctico para restituir los saberes locales como un acto de justicia (Santos, 2018) y proyectar una escuela futura desde los intereses de la comunidad en acción colectiva.

Durante los últimos años, en confluencia con estas directrices y en nuestro contexto se han desarrollado un amplio número de iniciativas emancipadoras, primando las estrategias de investigación-acción como una aproximación que permite una relación constante de práctica y reflexión sobre el quehacer propio de las instituciones escolares (Díaz-Bazo, 2017; Erausquin y Dome, 2016; Pérez, 2017). Destacan los enfoques centrados en la voz del estudiantado (Escobedo et al., 2017; Pacheco-Salazar, 2018; Yáñez-Urbina et al., 2018; Figueroa-Céspedes y Yáñez-Urbina, 2020); el trabajo con docentes en comunidades profesionales de aprendizaje (Beltrán et al., 2015; Guerra et al., 2017); y la indagación colaborativa como estrategia que involucra a toda la comunidad educativa (Moliner y Ramel, 2020; Moliner et al., 2020; Figueroa-Céspedes et al., 2016; Soto Cárcamo et al., 2017).

\subsection{La propuesta de la guía para la inclusión educativa}

Las directrices trazadas desde la noción de conocimiento emancipador encuentran un correlato y articulación práctica en la propuesta de la Guía para la Inclusión Educativa (Booth y Ainscow, 2012), correspondiente a un conjunto de materiales de apoyo a la autoevaluación de la escuela con miras al cambio educativo. El texto parte de la idea de que todos los agentes educativos poseen un conocimiento acerca de las mejoras necesarias que deben realizarse en sus escuelas y son sujetos activos en los procesos de cambio (Booth y Ainscow, 2012).

El material se inspira en una perspectiva de investigación-acción colaborativa y participativa (Figueroa-Céspedes y Muñoz, 2014), ya que orienta el desarrollo de procesos de autoexploración de las comunidades educativas a través de tres dimensiones analíticas: políticas, culturas y prácticas (Booth y Ainscow 2012). Siendo la primera vinculada a la gestión de la escuela y sus planes de cambio; mientras que la segunda enfatiza las relaciones, las creencias y los valores profundamente arraigados; y la tercera se basa en lo que se enseña y en cómo se enseña y aprende.

La concepción o idea de inclusión que se devela en los materiales proponen un nivel de agencia relevante de parte de los distintos colectivos, buscando construir conocimientos compartidos acerca de las formas en que la institución reconoce y trabaja las temáticas de la diversidad educativa, reconociendo barreras y recursos para la inclusión educativa. Los cambios que persigue la inclusión en educación, no pueden ser reducidos a una colección de innovaciones didácticas ni a una sumatoria de reformulaciones organizativas (Murillo, 2002), sino que deben orientarse a modificar las formas de pensar y actuar de la comunidad de modo tal de promover una mejora constante de los procesos y los resultados (Murillo y Krichesky, 2012). Lo anterior, implica transitar hacia una cultura escolar orientada al cambio y desarrollo, con la finalidad de garantizar el éxito de los esfuerzos por transformar la escuela.

Por lo tanto, el concepto de desarrollo inclusivo presentado en la Guía está ligado estrechamente a la puesta en acción de valores inclusivos tales como la participación, el respeto a la diversidad y la esperanza (Booth y Ainscow 2012) dando coherencia a las 
mejoras y transformaciones que realiza la escuela bajo una variedad de etiquetas (por ejemplo, educación en derechos, convivencia, educación ambiental, entre otros) a fin de fomentar el aprendizaje y la participación de niños/as y sus familias, el personal, el equipo directivo y demás miembros de la comunidad (Booth y Ainscow 2012).

Respecto a experiencias con la Guía, se ha señalado que el material resulta «sensible», dado que propende al debate $\mathrm{y}$, en ocasiones, al conflicto, el que, sin embargo, puede ser superado dependiendo de su abordaje en las escuelas, fomentando un buen nivel de apropiación de la propuesta técnica de los materiales (Durán et al., 2005; Education, Audiovisual \& Culture Executive Agency, 2012). Por otra parte, Figueroa-Céspedes y Muñoz (2014) analizaron el proceso de evaluación institucional haciendo uso de los materiales de la guía, concluyendo que, para los participantes, fue posible construir una visión reflexiva y transformadora de la inclusión, siendo vital la participación durante el proceso.

Con estos elementos, es menester ahora dar cuenta del método de investigación, caracterizando la experiencia desarrollada, para contextualizar nuestro ulterior análisis.

\section{Método}

La presente investigación de carácter cualitativo, corresponde a la sistematización de una serie de experiencias de asesoramiento colaborativo implementadas desde un diseño de investigación-acción participativa con las comunidades educativas (Becerra y Moya, 2010). Esta estrategia es coherente con el enfoque transformador presente en la propuesta de la Guía para la Inclusión Educativa (Booth y Ainscow, 2012).

En esta línea, el trabajo se desarrolló en el marco de un convenio colaborativo entre el Centro de Desarrollo Cognitivo de la Universidad Diego Portales y una comuna del sector norte de la ciudad de Santiago de Chile que administra las siete escuelas con las que llevaron a cabo las experiencias entre los años 2014 y 2017. En términos contextuales, el sistema de educación municipal de la comuna atiende los niveles de educación parvularia, básica y media contando con sistemas de apoyo y focalización que consideran las distintas dimensiones de vulnerabilidad y, en particular, orientando la entrega de recursos para financiar acciones de mejora, las que deben diseñarse tomando en consideración los desafíos de las evaluaciones estandarizadas como principal indicador de cambio.

Las técnicas de producción de información en este contexto involucraron observación participante y el registro de notas de campo (99 notas) de las sesiones registradas por los asesores colaborativos durante todo el periodo estudiado. Además, se incluyeron todos los materiales producidos por las comunidades educativas (actas de reuniones, documentos internos, material de divulgación a la comunidad, informes de evaluación y planes de acción), así como también entrevistas a los equipos de las escuelas participantes (14 entrevistas).

Sobre dicho material, se empleó un análisis de contenido (Cáceres, 2008) y una triangulación de datos (Fusch et al., 2018) con la finalidad de sistematizar categorías en torno a las claves para orientar procesos de desarrollo escolar inclusivo. Operativamente, se procedió a trabajar con el material de manera diferenciada en la construcción de dimensiones de tipo inductivo dando lugar a diversas producciones académicas, las cuales posteriormente fueron integradas a través de procesos de contraste entre las diversas escuelas y el tipo de actor involucrado en el asesoramiento educativo. Luego de lo cual se 
obtuvieron cinco dimensiones consideradas clave para los procesos de desarrollo escolar inclusivo (Figueroa-Céspedes et al., 2016), las que en esta investigación se profundizaron desde la mirada del conocimiento emancipador.

En términos procedimentales, la experiencia en los colegios siguió un ciclo de investigación-acción, en donde inicialmente se desarrolla una formación que da inicio al proceso, posteriormente los equipos exploran y analizan la realidad escolar, luego del cual elaboran un plan de desarrollo con orientación inclusiva, implementando y evaluando de forma continua, a partir de la gestión y seguimiento de un equipo coordinador, representativo, participativo y colegiado. En términos concretos, se realizó un trabajo en tres fases que sintetizamos a continuación:

\section{Fase 1. Inicial formativa}

Las primeras aproximaciones se desarrollaron por medio de jornadas formativas llevadas a cabo a finales del año 2014 e inicios del 2015. Esta actividad duró 24 horas cronológicas, divididas en ocho sesiones de trabajo que buscaban problematizar el desarrollo educativo en las escuelas, a partir del estudio de los materiales, la realización de reflexiones compartidas y casos coordinados por nuestro equipo. Algunas de las temáticas revisadas fueron:

- Educación en y para la diversidad

- Valores inclusivos en acción

- Culturas, políticas y prácticas inclusivas

- La investigación-acción como herramienta de transformación educativa

- Las barreras para el aprendizaje y la participación

- Los procesos de autoexploración dentro de la escuela

En cada sesión se desarrollaron actividades de facilitación y debate en grupo utilizando las propuestas de la guía, añadiendo algunas variaciones relevantes. Por ejemplo, se realizaron entrevistas y dinámicas de reconocimiento entre los participantes, algunas de ellas vinculadas a ejercicios de role-playing. Además, se utilizaron los cuestionarios y otras modalidades de exploración de la escuela (audiovisuales, fotografías, dibujos y similares).

\section{Fase 2. Auto-exploración}

Luego, se organizaron los equipos de coordinación por cada escuela, para durante el año escolar 2015 implementar el programa. Cada uno estuvo conformado por directivos, docentes, asistentes de la educación, estudiantes y sus familias, existiendo variaciones en la conformación de cada escuela, dependiendo de sus hábitos para trabajar en forma participativa y colegiada.

Asimismo, cada institución contó con el apoyo de un asesor colaborativo o "amigo crítico", como un profesional familiarizado con el material de la Guía y cuyo rol era orientar y colaborar en los procesos de investigación-acción, aportando preguntas y visiones alternativas que buscaban profundizar en la comprensión de las barreras detectadas.

Los equipos mantenían reuniones periódicas para poder realizar un proceso de autoexploración de la escuela por medio del material entregado. Así se desarrollaron diagnósticos participativos explorando las barreras y recursos a nivel de culturas, políticas y prácticas. Dicho proceso tenía como finalidad el diseño de un plan de acción orientado a 
dar respuestas a las problemáticas encontradas y ser ejecutado y evaluado durante el año 2016.

\section{Fase 3. Diseño e implementación}

Como resultado de algunas dinámicas internas que obstaculizaron el proceso y a la falta de experiencia en diagnósticos participativos reconocida por los establecimientos educativos, existieron dificultades en la fase 2. En función de ello, resultó necesario desarrollar un acompañamiento durante la primera parte de esta fase. De esta forma, las escuelas elaboraron un Plan de Desarrollo Inclusivo para abordar las barreras identificadas a partir de la definición de objetivos para cada una de las dimensiones.

No obstante, en la segunda parte, no todas las escuelas mantuvieron sus equipos operativos, en algunos casos se fusionaron con otros que la escuela ya tenía en funcionamiento y en otro simplemente fueron desapareciendo con el tiempo, dando lugar a un seguimiento por parte del equipo directivo o de su consejo escolar.

En esta línea, solo una de las escuelas desarrolló un proceso de re-evaluación de su Plan de Desarrollo Inclusivo contando con el apoyo de un amigo crítico durante el segundo semestre del año 2016. Proceso que se llevó a cabo por medio de un trabajo con el estudiantado que permitió indagar sobre las barreras al aprendizaje y la participación, utilizando herramientas audiovisuales (fotografías y dibujos) (Yáñez-Urbina et al., 2018). Gracias a dicha experiencia, la escuela en particular determinó la necesidad de replantear sus objetivos, transitando desde un foco puesto en el desarrollo del pensamiento crítico hacia prácticas críticas basadas en la comunidad.

\section{Resultados}

Considerando el análisis global de la experiencia, emergen una serie de dimensiones que favorecen la generación de condiciones para el desarrollo escolar inclusivo (FigueroaCéspedes et al., 2016), y que se examinan en este artículo a partir del conocimiento emancipador (Figueroa-Céspedes y Yáñez-Urbina, 2020). En cada dimensión se detallan tanto barreras como facilitadores sistematizados por el equipo investigador a partir de los procesos de indagación colaborativa llevados a cabo. A continuación, describimos cada una de ellas.

\subsection{Culturas colaborativas}

Esta primera dimensión, guarda relación con el proceso de construcción conjunta de los distintos agentes de la comunidad educativa a través de la colaboración. La experiencia desarrollada implicó abrir instancias de diálogo inéditas en la mayor parte de los establecimientos, conformándose equipos relativamente transversales, y operativos en cada escuela, aunque con continuidad y proyección variables.

Estas instancias fueron útiles para procesar y comprender lo que implica la inclusión en educación. Destacamos en esta dimensión la importancia de la co-responsabilidad asumida por los equipos en torno a la mejora de la escuela y la preexistencia de prácticas colaborativas. En esta dimensión resulta clave el fomento (directo o indirecto) de la participación activa y el diálogo abierto en los equipos, situación que genera el clima de confianza necesario para favorecer la negociación de significados y el disenso, en miras a concordar perspectivas en la producción e interpretación de la información sobre la escuela. Por otra parte, como barreras para el desarrollo de culturas colaborativas, en 
términos generales, en ciertos casos se pudo observar una tendencia a "tolerar" dicha participación valorando su existencia sólo desde una perspectiva burocrática y puntual. Una óptica que prima en este escenario es la asunción de falsos consensos y silenciamiento del disenso, aspecto sobre el cual resulta necesario ejercer un rol formativo y generador de condiciones en cada escuela.

Resulta clave considerar esta dimensión, en términos auténticos, para posibilitar la emergencia de un conocimiento emancipador en y sobre la escuela, en tanto apertura e intercambio entre la multiplicidad de perspectivas y lugares de enunciación dentro de la misma, por sobre su mera presencia.

\subsection{Investigación-acción participativa}

La segunda dimensión se refiere al valor de los procesos de indagación colaborativa y de reflexión sistemática en torno a la evidencia recolectada. Los procesos de autoexploración de la escuela, siguieron una lógica de investigación acción participativa, a través de los materiales de la Guía. En este sentido, aquellos equipos que asumieron el desafío consciente de investigar su propia escuela logran implementar metodologías de autoexploración y planificación pertinentes a su contexto.

Los equipos de trabajo valoraron la existencia de espacios y tiempos para la reflexión sistemática, la que se daba por lo general de forma limitada y acotada por las condicionantes institucionales. Una de las principales barreras detectadas durante el proceso es el débil reconocimiento de la necesidad de participación auténtica de toda la comunidad educativa y para el desarrollo de un proceso transformador. De esta manera, resultó un desafío trascender la lógica de la participación consultiva y aditiva a través del "cuestionario de indicadores" de la guía, aspecto que finalmente se abordó gracias a que los "amigos críticos" enfatizaron en la importancia de crear nuevos espacios de reflexión y de indagación colaborativa, más intensivos y con un nivel de participación más profunda.

Por otra parte, las dinámicas iniciales de resistencia de los directivos ante este ejercicio de análisis participativo, se relacionaban con sus dificultades para cuestionar sus propias predefiniciones y priorizaciones, y para resolver las tensiones emergentes al acceder a evidencia discordante arrojada por el diagnóstico participativo. Esto implicó una tendencia inicial a mantener la visión y predeterminación acerca de los temas que debían ser objeto de cambio en la escuela y que en general se condicen con la política (y burocracia) educativa vigente.

Esta situación se asociaba con la ilusión de participación que emerge a partir del uso preponderante de técnicas cerradas de producción de datos. Esto resultó problemático en particular en aquellas escuelas en donde la participación de los actores se caracterizaba por ser más puntual y restringida. También, debemos recalcar la experiencia desarrollada en dos escuelas a partir de la realización de propuestas innovadoras de autoexploración incorporando metodologías audiovisuales y narrativas, lo que permitió perfilar un mayor enriquecimiento del proyecto.

Resulta llamativo observar que, si bien los establecimientos deseaban construir propuestas más inclusivas, no lograban conectar inicialmente ese anhelo con metodologías acordes a ese fin, muchas veces tratando de imponer perspectivas hegemónicas. Entre estas, se puede mencionar uno de los casos en que se realizaron metodologías participativas con los estudiantes para recoger sus voces (lideradas por parte del equipo coordinador) las que posteriormente fueron cuestionadas por uno de los directivos por ser consideradas "poco 
representativas" y "sesgadas" en relación a los cuestionarios. Resulta necesario, por tanto, considerar que sobre la base de una indagación y construcción situada de conocimiento escolar - sea en modalidad de investigación acción u otra- es clave la apertura para incorporar y validar estrategias de indagación que demandan alta implicación en el proceso. Asimismo, es central que el equipo tenga disposición para redefinir los marcos interpretativos acerca del problema en deliberación, desde una perspectiva de justicia epistémica, con las implicancias políticas que esto conlleva.

\subsection{Liderazgo inclusivo}

Esta dimensión remite a las formas en que se expresa el liderazgo directivo y educativo. En aquellos establecimientos que delegaban y distribuían las responsabilidades a través de la conformación de equipos y que potenciaban la toma de decisiones colegiadas al interior de la unidad educativa, se gestaron interesantes procesos de cambio. De esta forma, a nuestro juicio, un liderazgo abierto, participativo y distribuido resulta esencial para orientar procesos de desarrollo inclusivo. Así, pudimos identificar algunos rasgos en los equipos directivos que potenciaron el proceso deliberativo, como la construcción de vínculos de confianza en los equipos, el fomento de un diálogo abierto, el desarrollo de prácticas de democracia interna y de escucha de las voces disidentes. Además, resultó relevante la delegación de tareas y el fomento de liderazgos emergentes en cada equipo.

Las barreras que afectaron el proceso se remiten esencialmente, a estilos de liderazgo tradicionales y de orden burocrático, caracterizados por un fuerte autoritarismo y la necesidad de supervisar y regular el trabajo del equipo coordinador, para disminuir el impacto potencial que la apertura a nuevas miradas pudiera generar a su gestión directiva. En este sentido, algunos establecimientos mostraron una tendencia a limitar la participación y a centralizar las decisiones finales. La construcción de un liderazgo con un posicionamiento ético, acorde a la mirada inclusiva, abierto al disenso, flexible, permite manejar la alta dosis de incertidumbre que emerge del trabajo de análisis de barreras y la toma de decisiones, potenciando la adherencia a un plan de desarrollo inclusivo construido colaborativamente.

Sin duda esta es una dimensión, estrechamente vinculada a las culturas colaborativas, que es condición necesaria y facilitadora de la adhesión y despliegue del proceso colaborativo y distribuido de construcción de conocimiento apropiado y común.

\subsection{Visión de cambio integral}

Esta dimensión tiene que ver con visualizar a la escuela en su complejidad y no de forma fragmentaria. En nuestra experiencia, algunas escuelas suelen seccionar su quehacer en distintas áreas que poco se conectan entre sí. Esta desconexión muchas veces está orientada por el currículum y la política educativa tradicional, pero también recae en una cultura que segmenta para abarcar la complejidad pedagógica. Un principio que trabajamos en nuestra experiencia es la necesidad de fomentar una visión sistémica de la escuela, apelando al desarrollo de sinergias programáticas entre distintas iniciativas que conviven en la escuela y que comparten principios pedagógicos similares.

Esta orientación nos mostró un camino interesante por el cual transitamos en nuestro proyecto. Una de las ideas fuerza que se trabajaron, fue el hecho de que tradicionalmente ante problemas y desafíos complejos se buscan respuestas simplistas y parciales. Por ejemplo, en algunas escuelas los equipos buscaron la articulación del proyecto con iniciativas afines preexistentes o ejecutadas de forma simultánea (por ejemplo, acciones en 
convivencia, proyectos comunitarios), y específicamente con los instrumentos de gestión institucional (Proyecto Educativo Institucional, por ejemplo).

No obstante, la falta de una visión integral de la escuela resulta ser una de las dificultades a las que suelen estar enfrentadas las instituciones. Las diferentes demandas propias de la cotidianeidad escolar y de sus variables político-administrativas, generan una fragmentación de acciones muy compleja de reorientar. La necesidad de dar respuesta y la falta de condiciones para la colaboración configuran características que impiden a los equipos superar la visión de la escuela como un conjunto de programas aislados. Al respecto, en algunos casos las propuestas de cambio generadas por los equipos corrieron el riesgo de ser sustituidas por definiciones previas surgidas de instancias externas a la propia escuela y que, por distintas razones, se trataron de imponer bajo el pretexto de generar sinergias con otros programas de la escuela.

Esta tendencia, fue un problema que requirió de parte del "amigo crítico" la realización de una interpelación más directa hacia las autoridades, lo que permitió regular dichas intenciones y revertir, en cierto grado, la intención original. Esta dimensión implicó la necesidad de remirar el concepto de desarrollo o mejora escolar que manejan las escuelas y cuestionar su base ligada al gerencialismo y su agenda de estándares. Fue relevante reconocer que la vida escolar es compleja e incluye múltiples formas de ser desarrollada. En este escenario, la mirada de los "valores en la acción" constituye un buen recurso para interrogar los enfoques de desarrollo y cambio que están a la base de este ejercicio. Considerar la escuela como un todo complejo, frente a la lógica de la fragmentación, implicó desde la propuesta de trabajo, reconocer la coexistencia de ambos enfoques, apuntando a abordar las potenciales pugnas respecto del sentido de la escuela, provocando un cuestionamiento a su tradición monocultural.

\subsection{Asesoramiento colaborativo}

Esta dimensión alude al enfoque del asesoramiento llevado a cabo. Consideramos relevante en este proceso orientar y potenciar la autonomía reflexiva de los equipos, a través de la colaboración con el otro, aspecto que se constituyó en una dimensión crucial en este proceso. En este sentido, la modalidad de trabajo desde el "amigo crítico" fue bien valorada por los participantes, no obstante existieron desacomodos iniciales, vinculados a lo inédito de un rol de este tipo en los establecimientos. Este asesoramiento, permitió movilizar conocimientos a partir de la práctica de la investigación acción, gestando condiciones e interpelando al liderazgo directivo, con el propósito de crear mayor consciencia respecto a la relevancia de construir culturas colaborativas, desde una visión compleja e integral sobre la escuela.

Entre los elementos favorecedores destacamos el rol de un asesoramiento comprometido y facilitador, que contribuye a validar las voces de todos los actores, a la vez que favorece la reflexión y la interrogación de las dinámicas regulares de la escuela, fomentando la conexión constante de las acciones con valores inclusivos. Este proceso en las escuelas resultó ser extremadamente desafiante, ya que implicó la necesidad de renegociar el rol del asesor entre un participante más directivo, que genera condiciones mínimas para que emerja el conocimiento-emancipador, y el de un observador participante, que acompaña y aporta nuevas perspectivas a dicha construcción. Esto necesariamente nos hace preguntarnos acerca del lugar del acompañamiento en los procesos de cambio, ¿cómo diseñar metodologías de asesoramiento que respondan a este justo equilibrio que implica la indagación colaborativa?. En cualquier caso, cabe señalar que el desmarque de una 
posición de experto del asesor colaborativo, contribuye a la validación de los actores como a la co-construcción del conocimiento respecto de la escuela.

Finalmente, la experiencia analizada nos muestra que estas dimensiones, al ser potenciadas por las instituciones, generan condiciones para la emergencia de una mirada emancipadora, orientada por el desafío de construir una escuela abierta a la diversidad. De esta forma, tal como hemos podido apreciar, estas dimensiones no constituyen células aisladas, sino que operan articuladamente para propiciar el desarrollo escolar inclusivo (Figura 1).

\section{Figura 1}

\section{Dimensiones clave del desarrollo escolar inclusivo}

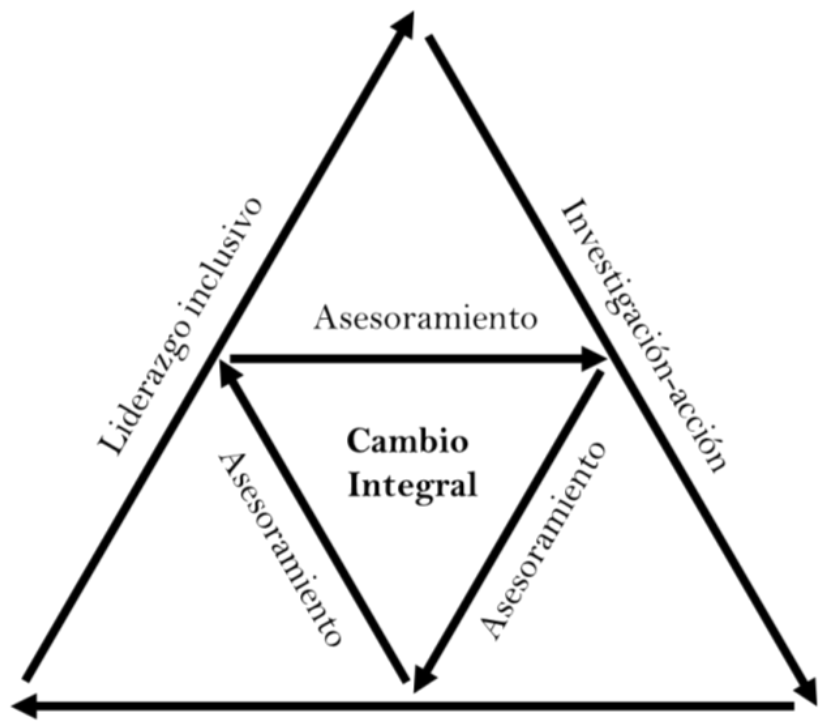

Culturas colaborativas

\section{Discusión y conclusiones}

En el presente trabajo abordamos cinco dimensiones clave para construir procesos de desarrollo escolar inclusivo en un contexto marcado por la hibridez y coexistencia entre un enfoque centrado en la estandarización de resultados y, por otro, enfocado en la valoración de la diversidad. En tal sentido, cada una de las dimensiones se encuentra permeada por la ambigüedad paradigmática de la política educativa, pudiendo variar entre abordajes eficientes, burocráticos, integrativos o comunitarios de acuerdo con las posibles combinaciones (Figueroa-Céspedes et al., 2019).

Lo anterior, deja en evidencia la constante dificultad que existe para articular estas dimensiones, al ser influidas por una amplia gama de factores. No obstante, la experiencia reportada también deja patente que, a pesar de las contradicciones respecto a las exigencias a las cuales son sometidas las escuelas, resulta importante des-centrar la figura de los "resultados" haciendo que prime un enfoque centrado más en los procesos. En otras palabras, es posible llevar a cabo transformaciones de las instituciones escolares siempre y cuando se ponga bajo examen aquellas condiciones que impidan el desarrollo de culturas, políticas y prácticas inclusivas.

En esta línea, si bien los estándares impuestos a las escuelas delimitan un terreno de acción y una compulsión por ofrecer respuestas que les permita obtener la etiqueta de "calidad", 
las estrategias por medio de las cuales se puede cumplir dicho objetivo pueden ser múltiples de acuerdo a las características y necesidades propias de cada establecimiento. Entonces, una mirada que priorice el producto por sobre el proceso, desplaza factores tales como la diversificación curricular, la participación de la comunidad, la convivencia escolar, la gestión de recursos, entre otros que en mayor o menor medida tienen un impacto en cómo se consolidan los procesos inclusivos.

Frente a este escenario, las experiencias analizadas permiten concluir que es posible cuestionar las formas en que se desarrollan las escuelas (Ainscow, 2005). Para esto, es imperioso fomentar el desarrollo de dispositivos pedagógicos que recursivamente analicen y transformen sus contextos, propiciando así el pensamiento crítico, la reflexión transversal, y los entornos activos para el aprendizaje y la participación de todos los miembros de las comunidades educativas. En este sentido, los resultados de nuestra indagación concuerdan con los estudios de Durán y otros (2005) y la EACEA (2012), en el sentido que la guía para la inclusión es un material "sensible", puesto que genera un debate abierto sobre la escuela, cuestionando sus finalidades y sentidos. Lo anterior implica que estas autoexploraciones requieren de diálogo honesto, de una negociación de significados, en los que muy posiblemente emerja conflictividad, en términos de intercambio de perspectivas, ante lo cual resulta relevante contar con un enfoque epistemológico situado y con apoyos colaborativos, como los aportados por los "amigos críticos".

Por otro lado, siguiendo a Moliner y St. Vincent (2014), consideramos que la investigación situada puede transformarse en una forma de revitalizar la democracia y la justicia social en las escuelas, articulando una ética que posicione como protagonistas a la comunidad educativa relevando sus voces y perspectivas para generar conocimiento significativo para la institución. En particular, nos interesa producir una sinergia en términos de los objetivos que tiene la investigación-acción participativa, con un foco puesto en producir cambios en la institución y una investigación de corte académico, con una orientación hacia la divulgación científica.

Siendo así, tal como planteamos en la dimensión de asesoramiento colaborativo y como nos exige un marco del conocimiento emancipador, es necesario volcar los esfuerzos hacia un quehacer en conjunto y dialógico (Long, 2007), que no aspire a encontrar en los equipos externos una verdad (Haraway, 2004) respecto a las dinámicas internas o una representación de mundo (Haraway, 1999), sino que tenga como norte el desarrollo de una comunidad investigativa autónoma y crítica ante sus propios procesos orientada hacia la transformación y mejora educativa (Soto Cárcamo et al., 2017).

En este sentido, en la experiencia llevada a cabo se torna clave la construcción de culturas que consideren la diversidad propia de sus comunidades y rechacen la noción monoculturalista de la escuela (Booth y Ainscow, 2012; Dietz, 2012). Así, el proceso de asesoramiento realizado buscó volver inteligible (Butler, 2006) aquellas voces subalternas silenciadas (Spivak, 2011) y, en un ejercicio de justicia, recobrar las experiencias y los conocimientos locales que ellas aportan (Santos, 2018) para instalar procesos deliberativos basados en una objetividad consensuada comunitariamente (Haraway, 1995).

No obstante, hemos advertido que es necesaria una mirada atenta a aquellos intentos de imposición de una perspectiva hegemónica sobre el propio ejercicio de la escuela, en donde los liderazgos tradicionales y/o autoritarios tienden a cooptar para sí la posibilidad de producir un conocimiento válido sobre la escuela y sus proyecciones futuras. Es posible 
considerar estas prácticas como ejercicios que buscan la conservación de un orden imperante (Chavarría y García, 2004), trampas que dificultan el reconocimiento de los actores propiciando jerarquías y señalando que solamente existe una forma válida de producir conocimiento en la escuela.

Esta tensión, nos lleva a mirar la polisemia del concepto inclusión y la diversidad de formas que tienen los distintos actores de representarse el cambio educativo (FigueroaCéspedes et al., 2019), al tiempo que darle relevancia al hecho de propiciar culturas de cambio en la escuela (Fullan, 2002; Murillo y Krichesky, 2012) con miras a la justicia social. Por lo tanto, resulta necesario construir una noción de cambio comunitario y emancipador (Figueroa-Céspedes et al., 2019), contando con apoyos desde una perspectiva crítica (Soto Cárcamo et al., 2017).

Ahora bien, otro componente que debemos considerar es la micropolítica del cambio, pues en las experiencias de mejora observadas fue necesario gestionar condiciones mínimas de operación: tiempos, recursos y espacios, implicando en algunos casos tensiones para el desarrollo de nuestra propuesta de amistad crítica. Lo anterior, debido a que las negociaciones para lograr los mínimos generaban desacuerdos entre el "amigo crítico" y los equipos directivos. En este sentido, la función de un liderazgo inclusivo representado por la dirección de cada establecimiento y el municipio se constituye en un factor clave para el desarrollo escolar en el marco de las oportunidades y limitaciones que tiene la política educativa a nivel central.

Siendo así, es fundamental continuar con la labor investigativa en torno a los procesos de desarrollo escolar inclusivo como una práctica de resistencia ante las lógicas imperantes en los sistemas educacionales, pues no solamente implica una labor orientada a crear otras formas de construir sociedad desde un rol político de la escuela (Dussel, 2004), sino que instala una crítica a un sistema de mejora escolar centrado en indicadores como únicos referentes de la calidad educativa, dejando de lado los mundos de vida y las perspectivas de los actores de la comunidad educativa.

No obstante, lo anterior también pone de manifiesto la imperiosa necesidad de desarrollar propuestas que no solamente se limiten al trabajo con docentes y el estudiantado. Siendo la escuela un espacio polimorfo y múltiple, resulta relevante considerar su ecología de desarrollo y fomentar la co-construcción de conocimientos con participación activa de los "otros" miembros de la comunidad educativa: las familias, asistentes de la educación, la comunidad "vecina" a la escuela, colectivos "disidentes", etc.

En suma, es necesario considerar la inclusión educativa no como una meta a lograr, sino como un proceso continuo de transformación asociado al ejercicio de movilizar (y por qué no decirlo, democratizar) el conocimiento (Moliner y Ramel, 2020) para, de esta forma, abordar las barreras que imposibilitan que todos los actores participen, aprendan y se desarrollen en una escuela común.

\section{Referencias}

Abramovay, M. (2005). Violencia en las escuelas: un gran desafío. Revista Iberoamericana de Educación, (15), 53-66. https://doi.org/10.35362/rie380830.

Ainscow, M. (2005). Developing inclusive education systems: what are the levers for change? Journal of Educational Change, 6(2), 109-124. https://doi.org/10.1007/s 10833-005-1298-4. 
Apablaza, M. (2018). Inclusión escolar, marginación y apartheid ocupacional: Análisis de las políticas educativas Chilenas. Journal of Occupational Science, 25(4), 3-15. https://doi.org/10.1080/14427591.2018.1487260.

Becerra, R. y Moya, A. (2010). Investigación-acción participativa, crítica y transformadora. Un proceso permanente de construcción. Revista Integra Educativa, 3(2), 133-156.

Beltrán, Y., Martínez, Y. y Torrado, O. (2015). Creación de una comunidad de aprendizaje: Una experiencia de educación inclusiva en Colombia. Revista Encuentros, 13(2), 57-72. https://doi.org/10.15665/re.v13i2.498.

Booth, T. (2005). Keeping the future alive: Putting inclusive values into action. Forum: For Comprehensive Education, 47(2), 151-158. https://doi.org/10.2304/forum.2005.47.2.4.

Booth, T., y Ainscow, M. (2012). La guía para la inclusión educativa. Fundación Creando Futuro.

Butler, J. (2006). Deshacer el género. Barcelona: Paidós.

Cáceres, P. (2008). Análisis cualitativo de contenido: Una alternativa metodológica alcanzable. Psicoperspectivas, 2(1), 53-82. https://doi.org/10.5027/psicoperspectivas-Vol2-Issue1-fulltext-3.

Calderón, D. (2015). Los niños como sujetos sociales: Notas sobre la antropología de la infancia. Nueva Antropol, 28(2), 125-140. http://www.scielo.org.mx/pdf/na/v28n82/v28n82a7.pdf.

Chavarría, M. y García, F. (2004) Otra globalización es posible. Diálogo con Boaventura de Sousa Santos. Iconos, 19, 100-111. https://doi.org/10.17141/iconos.19.2004.42.

Díaz-Bazo. C. (2017). La investigación-acción en la educación básica iberoamericana. Una revisión de literatura. Magis, 10(20), 159-182. https://doi.org/0.11144/javeriana.m10-20.iaeb.

Dietz, G. (2012). Multiculturalismo, interculturalidad y diversidad en educación: Una aproximación antropológica. Fondo de Cultura Económica.

Duarte, C. (2012). Sociedades adultocéntricas: Sobre sus orígenes y reproducción. Última década, 20(36), 99-125. https://doi.org/10.4067/s0718-22362012000100005.

Durán, D., Echeita, G., Giné, C., Miquel, E., Ruiz, C. y Sandoval, M. (2005). Primeras experiencias de uso de la guía para la evaluación y mejora de la educación inclusiva en el estado español. REICE. Revista Iberoamericana sobre Calidad, Eficacia y Cambio en Educación, 3(1), 464-467.

Dussel, I. (2004). Inclusión y exclusión en la escuela postestructuralista. Cuadernos de Pesquisa, 34(122), 305-335. https://doi.org/10.1590/S0100-15742004000200003.

Education, Audiovisual \& Culture Executive Agency. (2012). Analysis of the use and value of the index for inclusion (Booth $\mathcal{E}^{2}$ Ainscow, 2011) and other instruments to assess and develop inclusive education practice in P2I partner countries. Fontys.

Erausquin, C. y Dome, C. (2016). La investigación-acción como estrategia para re-visitar experiencias, posicionamientos y prácticas ante violencias en escuelas. Anuarios de Investigación, 23, 87-96.

Escobedo, P., Sales, A. y Traver, J. (2017). La voz del alumnado: Su silencio y la cultura profesionalista. Educación XXI, 2O(2), 299-318. https://doi.org/10.5944/educxx 1.19045

Ferrão, V. (2010). Educación intercultural en América Latina: Distintas concepciones y tensiones actuales. Estudios Pedagógicos, 36(2), 333-342. https://doi.org/10.4067/s07 18-07052010000200019.

Figueroa-Céspedes, I. y Muñoz, Y. (2014). La guía para la inclusión educativa como herramienta de autoevaluación institucional: Reporte de una experiencia. Revista Latinoamericana de Educación Inclusiva, 8(2), 179-198. 
Figueroa-Céspedes, I., Soto Cárcamo, J. y Sciolla Hapke, B. (2016). Dinámicas de recepción, apropiación y contextualización del enfoque de la guía para la inclusión educativa en escuelas municipales de una comuna de la región metropolitana. Pensamiento Educativo: Revista de Investigación Educacional Latinoamericana, 53(1), 1-13. https://doi.org/10.7764/pel.53.1.2016.4.

Figueroa-Céspedes, I., Soto Cárcamo, J. y Yáñez-Urbina, C. (2019). Concepciones sobre el cambio educativo en docentes de escuelas municipales participantes de un proyecto de desarrollo inclusivo. Revista Educación, 43(1), 380-399. https://doi.org/10.15517/revedu.v43i1.31297

Figueroa-Céspedes, I. y Yáñez-Urbina, C. (2020). Voces y culturas estudiantiles en la escuela: Una reflexión teórico-metodológica desde procesos de autoexploración de barreras a la inclusión educativa. Revista Latinoamericana de Educación Inclusiva, 14(1), 109-126.

Fullan, M. (2002). El significado del cambio educativo: Un cuarto de siglo de aprendizaje. Profesorado. Revista de Currículum y Formación del Profesorado, 6(1), 1-14.

Fusch, P., Fusch, G. y Ness, L. (2018). Denzin’s paradigm shift: Revisiting triangulation in qualitative research. Journal of social change, 10(1), 19-32. https://doi.org/10.5590/JOSC.2018.10.1.02

Galaz, A., Jiménez-Vásquez, M. y Díaz-Barriga, A. (2019). Evaluación del desempeño docente en Chile y México. Antecedentes, convergencias y consecuencias de una política global de estandarización. Perfiles Educativos, 41(163), 177-199. https://doi.org/10.22201/iisue.24486167e.2019.163.58935.

García Canclini, N. (2007). De cómo la interculturalidad global debilita al relativismo. En A. Giglia, C. Garma y A. de Teresa (Comps.), ¿̇Adónde va la antropología? (pp. 39-59). UAM.

Guerra, P., Figueroa-Céspedes, I., Salas, N., Arévalo, R. y Morales, A. (2017). Desarrollo profesional en educadoras de párvulo: Análisis de una experiencia formativa desde la investigación-acción y la interacción mediada. Estudios Pedagógicos, 43(3), 175-192. https://doi.org/10.4067/s0718-07052017000300010.

Hall, S. (2006). Estudios culturales: Dos paradigmas. Revista Colombiana de Sociología, 27, 233-254. https://doi.org/10.15446/rcs.

Haraway, D. (1995). Ciencia, cyborg y mujeres. La reinvención de la naturaleza. Ediciones Cátedra.

Haraway, D. (1999). La promesa de los monstruos: una política regeneradora para otros inapropiados/bles. Política y Sociedad, 30, 121-163.

Haraway, D. (2004). Testigo_modesto@segundo_milenio. Hombrehembra(c)_conoce_oncoratón(r). Editorial UOC.

Herrera, J., Reyes-Jedlicki, L. y Ruiz, C. (2018). Escuelas gobernadas por resultados: Efectividad escolar y políticas educacionales de la transición democrática, Chile 1990-2017. Psicoperspectivas, 17(2), 1-12.

https://doi.org/10.5027/psicoperspectivas-Vol17-Issue2-fulltext-1110.

Jorquera, C. (2015). La estructura escolar frente a los desafíos de la diversidad: Una mirada subalterna al problema de la educación inclusiva. Liminales. Escritos sobre psicología y sociedad, I(8), 35-43. https://doi.org/10.54255/lim.vol4.num08.269.

Long, N. (2007). Sociología del desarrollo: Una perspectiva centrada en el actor. Ciesas

Moliner, O. y St-Vincent, L. (2014). Dilemas éticos de los investigadores que acompañan procesos de investigación-acción, en el marco de la escuela intercultural inclusiva. Revista Latinoamericana de Educación Inclusiva, 8(2), 49-68.

http://www.rinace.net/rlei/numeros/vol8-num2/art2.pdf. 
Moliner, O. y Ramel, S. (2020). Procesos de movilización del conocimiento sobre educación inclusiva en las escuelas suizas. ¿Qué papel juegan los investigadores/formadores que asesoran los procesos de mejora escolar?. Estudios sobre Educación, 38(1), 169-189. https://doi.org/10.15581/004.38.169-189.

Moliner, O., Lozano, M. y Doménech, A. (2020). La construcción de un modelo participativo de acompañamiento a centros educativos para movilizar el conocimiento sobre la educación inclusiva. En O. Moliner (Coord.), Acompañar la inclusión escolar (pp. 19-32). Dykinson. https://doi.org/10.2307/j.ctv153k44b.6

Murillo, F. J. (2002). La mejora de la escuela: Concepto y caracterización. En F. J, Murillo y M. Muñoz-Repiso (Coords.), La mejora de la escuela: Un cambio de mirada (pp. 15-51). Octaedro.

Murillo, F. J. y Krichesky, G. (2012). El proceso de cambio escolar. Una guía para impulsar y sostener la mejora de las escuelas. REICE. Revista Iberoamericana sobre Calidad, Eficacia y Cambio en Educación, 10(1), 26-43.

Pacheco-Salazar, B. (2018). Las voces del estudiantado en la investigación socio-educativa: Trascendiendo el adultocentrismo. Ciencia y Educación, 2(2), 42-51. https://doi.org/10.22206/cyed.2018.v2i2.pp43-51.

Pérez, M. (2017). Investigación acción participativa (IAP) como elemento de fortalecimiento educativo en la escuela bolivariana "el paramito", estado de Mérida. Revista Scientific, 2(3), 223-242. https://doi.org/10.29394/scientific.issn.2542-2987.2017.2.4.13.223-242.

Rosas. K. (2012). Genealogía de los estudios culturales. Razón y Palabra, 81, 1-22.

Santos, B. D. S. (2018). Introducción a las epistemologías del sur. En M. Meneses y K. Bidaseca (Coords.), Epistemologías del sur (pp. 25-62). CLACSO. https://doi.org/10.2307/j.ctvnpok5d.4

Sisto, V. (2020). La escuela abandonada a evaluaciones y estándares, confinada en el managerialismo. Praxis Educativa, 15, 1-26.

https://doi.org/10.5212/PraxEduc.v.15.15777.107.

Soto Cárcamo, J., Figueroa-Céspedes, I. y Yáñez-Urbina, C. (2017). Asesoramiento colaborativo en escuelas municipales: Posicionamientos y desafíos del rol de amigo crítico en un proyecto de desarrollo escolar inclusivo. Revista Latinoamericana de Educación Inclusiva, 11(1), 245264. https://doi.org/10.4067/s0718-73782017000100015.

Spivak, G. (2011). ¿Puede hablar el subalterno?. El Cuenco de Plata

Stoll, L. y D. Fink (1999). Para cambiar nuestras escuelas. Reunir la eficacia y la mejora. Octaedro.

Tomé, M. y Manzano, B. (2016). La educación inclusiva intercultural en Latinoamericana. Análisis legislativo. Revista Nacional e Internacional de Educación Inclusiva, 9(2), 1-17.

Urbina, C., Basualto, P., Durán, C. y Miranda, P. (2017). Prácticas de co-docencia: El caso de una dupla en el marco del programa de integración escolar en Chile. Estudios Pedagógicos, 43(2), 355-374. https://doi.org/10.4067/s0718-07052017000200019.

Yañez-Urbina, C., Figueroa-Céspedes, I., Soto Cárcamo, J. y Sciolla Hapke, B. (2018). La voz en la mirada: Fotovoz como una metodología para explorar los procesos de inclusión-exclusión desde la perspectiva del estudiantado. Pensamiento Educativo, 55(2), 1-16.

https://doi.org/10.7764/pel.55.2.2018.4. 


\section{Breve CV de los autores}

\section{Ignacio Figueroa-Céspedes}

Psicólogo y Licenciado en Psicología, Universidad de Santiago de Chile. Doctor (c) en Educación, Universidad Metropolitana de Ciencias de la Educación. Magister en Desarrollo Cognitivo con mención en evaluación dinámica de la propensión al aprendizaje, Universidad Diego Portales. Se desempeña como académico y coordinador de formación continua del Centro de Desarrollo Cognitivo de la Universidad Diego Portales, realizando docencia y guía de tesis en programas de postgrado y formación continua de la misma casa de estudios. Es docente de la cátedra Diseño de un proyecto de investigación acción y Seminario de título en la carrera de Educación Diferencial de la UDP. Sus líneas de investigación se centran en procesos de desarrollo inclusivo en instituciones educativas, aprendizaje profesional docente e interacciones pedagógicas. Email: ignacio.figueroa@mail.udp.cl

ORCID ID: http://orcid.org/O000-0002-2756-1831

\section{Jorge Soto Cárcamo}

Sociólogo, Universidad de la Frontera. Máster en Investigación en Sociología Aplicada, Universidad Autónoma de Barcelona. Actualmente cursa el Doctorado en Ciencias Sociales en la Universidad de Buenos Aires. Es coordinador de titulación del Magister en Desarrollo Cognitivo del Centro de Desarrollo Cognitivo de la Universidad Diego Portales, donde conforma el comité académico y dicta clases en los módulos de metodología cualitativa y taller de tesis 1 y 2 , además de la conducción de tesis en el mismo programa. Ha desarrollado docencia en educación superior en pre y postgrado en materias de sociología de la educación, educación intercultural y metodología cualitativa de la investigación. Posee experiencia en estudios y consultorías para agencias estatales y organismos internacionales, proyectos de investigación, formación continua y asesoramiento escolar. Sus líneas de investigación se relacionan con educación intercultural, inclusión educativa y educación y territorio. Email: jorge.sotoc@mail.udp.cl

ORCID ID: http://orcid.org/0000-0001-5107-5668

\section{Cristopher Yáñez-Urbina}

Psicólogo y Licenciado en Psicología de la Universidad de Santiago de Chile. Magister en Comunicación Política del Instituto de la Comunicación e Imagen de la Universidad de Chile. Actualmente es doctorando en Psicología y Transformaciones Sociales de la Pontificia Universidad Católica de Valparaíso. Trabaja como coordinador de Investigación y Publicaciones del Centro de Desarrollo Cognitivo de la Universidad Diego Portales y desempeña labores como profesor-ayudante en el Magister en Desarrollo Cognitivo de la misma casa de estudios. Además, es docente de Seminario de título en Educación Diferencial. Sus líneas de investigación se centran en el desarrollo de metodologías participativas en contextos de investigación acción-participativa, estudios críticos sobre infancia, y educación y sexualidad. Email: cristopher.yanez@mail.udp.cl

ORCID ID: http://orcid.org/0000-0001-7171-9686 